\title{
Comparative Study of Nerve Conduction Velocity Studies in Type II Diabetes Mellitus with \& Without Features of Clinical Peripheral Neuropathy
}

\author{
Nilesh Bhange ${ }^{1}$, Prachi Vaidya ${ }^{2}$ \\ ${ }^{1}$ Resident in Nephrology, MGM Medical College, Aurangabad, Maharashtra, India \\ ${ }^{2}$ Resident in Medicine, MGM Medical College, Aurangabad, Maharashtra, India
}

\begin{abstract}
Aim of our study was to study \& compare nerve conduction velocity (NCV) in the patients of Type II Diabetes Mellitus with \& without clinical peripheral neuropathy. Study population included DM type II patients. They were evaluated for Clinical Peripheral Neuropathy with the help of Neuropathy Symptoms Score \& Neuropathy Disability Score. On the basis of these scores they were divided into two Group A (CPN) - 50 Type II Diabetes Mellitus patients with features of Clinical Peripheral Neuropathy. Group B (WCPN) - 50 Type II Diabetes Mellitus patients without features of Clinical Peripheral Neuropathy. Then these Groups were subjected to Nerve Conduction Velocity Study. The most common symptom of neuropathy was Tingling, Numbness and most common sign was loss of Ankle Jerk. Distal symmetrical sensory neuropathy (DSSN) was major clinical type of neuropathy. In Group A patients, NCV study was abnormal in $90 \%$ patients. In Group B patients, NCV study was abnormal in 60\% patients. Most common type of neuropathy observed in NCV study was Axonal Sensorimotor type.
\end{abstract}

Keywords: Nerve Conduction Velocity, Diabetes Mellitus, Peripheral Neuropathy

\section{Introduction}

Diabetes mellitus (DM) is one of the most widespread chronic diseases in the world Prevention and early detection of complication in diabetic patients is crucial, especially in light of the estimated increase in worldwide diabetes to approximately 366 million by $2030 .^{1} \mathrm{DM}$ has two types of complications: microvascular and macrovascular. One of the most frequently-occurring microvascular complications is diabetic neuropathy, of which the most common type is distal symmetrical neuropathy or peripheral neuropathy $(\mathrm{PN}){ }^{2}$ Incidences of diabetic PN have been reported in $10-$ $50 \%$ of patients with diabetes mellitus in Western countries. ${ }^{3}$ At the time of diagnosis, $\mathrm{PN}$ is present in $10 \%$ of diabetic patients and overall in $50 \%$ of patients with a 25 -year history of the disease. ${ }^{4}$

Peripheral neuropathy is the first step in the generation of diabetic foot ulcer. Foot ulcers develop in risk areas that are exact pressure points. If not detected early, these lesions may progress to gangrene and result in amputation. ${ }^{5,6}$

We conducted this study with aim to study \& compare NCV studies in the patients of Type II Diabetes Mellitus with \& without Clinical Peripheral Neuropathy. Objectives of our study were to study clinical profile of Peripheral Neuropathy in Type II Diabetes Mellitus patients with the help of Neuropathy Symptoms Score (NSS), Neuropathy Disability Score (NDS). We also studied NCV studies in Type II Diabetes Mellitus patients \& compared clinical and NCV study profile in the patients of Type II Diabetes Mellitus with \& without Clinical Peripheral Neuropathy.

\section{Methodology}

Study population included Diabetes Mellitus patients. The diagnosis of type II Diabetes Mellitus was done according to the criteria laid down by American Diabetes Association 2013 . $^{7}$ Then those patients who had associated conditions which can cause Peripheral Neuropathy were excluded from the study. Then all the patients were divided into two groups on basis of presence of Clinical Peripheral Neuropathy. Presence of Clinical Peripheral Neuropathy was evaluated with help of Neuropathy Symptoms Score (NSS) was evaluated by presence of neuropathic pain or paraesthesia. The calculation was done as - Present-1, Absent - 0. A score of 2 or more was taken as abnormal.

Neuropathy Disability Score (NDS) was evaluated by the presence of deep tendon reflexes, Vibration perception, pain sensation, touch sensation, temperature sensation. Responses: - Normal - 0, Decreased -1, Absent- 2. A score of 2 or more was taken as abnormal.

Depending upon results, 2 groups were made. Both groups had 50 patients. These Groups were

a) 50 Type II Diabetes Mellitus patients with features of Clinical Peripheral Neuropathy (CPN)

b) 50 Type II Diabetes Mellitus patients without features of Clinical Peripheral Neuropathy (WCPN)

\section{Results}

In our study, CPN group had higher number of patients having diabetes duration of 5 years to $>5$ years \& lesser number of patients having newly detected diabetes as compared to WCPN group. Most common symptom noted was Tingling \& Numbness $(100 \%) \&$ among the physical signs, Ankle jerk was found to be lost in $20 \%$ patients. 


\section{International Journal of Science and Research (IJSR) \\ ISSN (Online): 2319-7064}

Index Copernicus Value (2016): 79.57 | Impact Factor (2015): 6.391

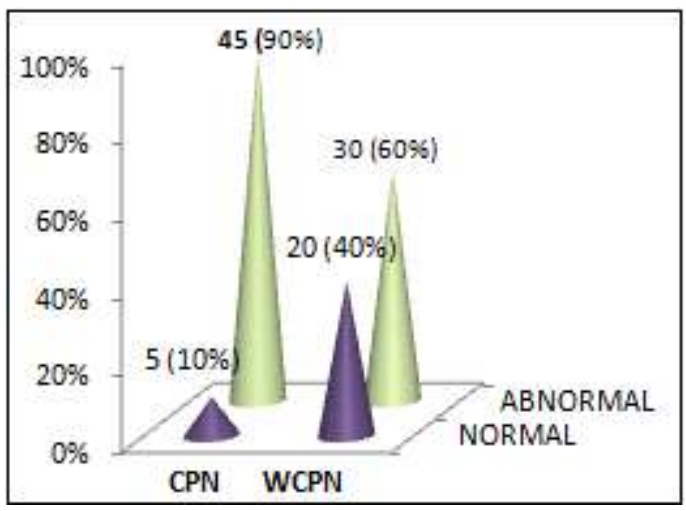

Figure 1: Clinical Neuropathy \& NCS relation

Distal symmetrical sensory neuropathy (DSSN) was major clinical type of neuropathy accounting for $86 \%$, followed by sensorimotor neuropathy (SMN) 10\%. Among CPN patients, $90 \%$ patients showed abnormal NCS report while 10\% patients showed normal NCS report. Among WCPN patients, $60 \%$ patients showed abnormal NCS report while $40 \%$ patients showed normal NCS report. Most common type of neuropathy observed on NCS was axonal sensory motor type (in 30\% patients).

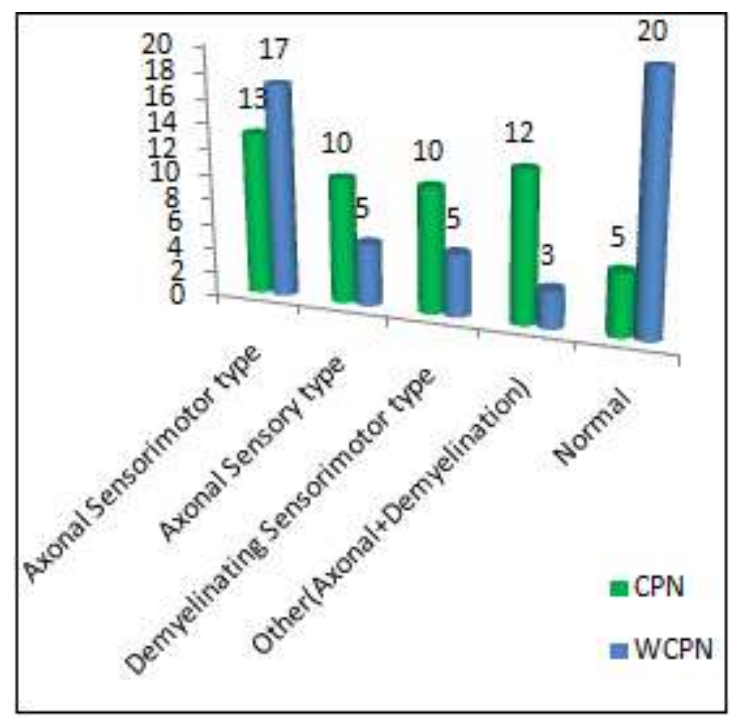

Figure 2: Details of NCS report

\section{Discussion}

In our study, most common symptom noted was Tingling \& Numbness (100\%). Among the physical signs, Ankle jerk was found to be lost in $20 \%$ patients. These findings were consistent with one study by Dyck PJ et $\mathrm{al}^{8}$ which observed that $90 \%$ patients had sensory symptoms with loss of vibration sense as the most common sign in $27 \%$ patients. Among the CPN patients, the most common presentation observed was distal symmetrical sensory neuropathy $(86 \%)$. It was comparable to a study by AL kakrani et al ${ }^{9}$ who also noted that distal symmetrical sensory neuropathy was the most common clinical type (84\%) noted. Out of the 100 patients studied, reports of NCS revealed $75 \%$ abnormal studies while $25 \%$ showed normal studies. This was almost similar to one study by Dyck PJ et $\mathrm{al}^{8}$ in which abnormal NCV studies were present in $72 \%$ of the patients.

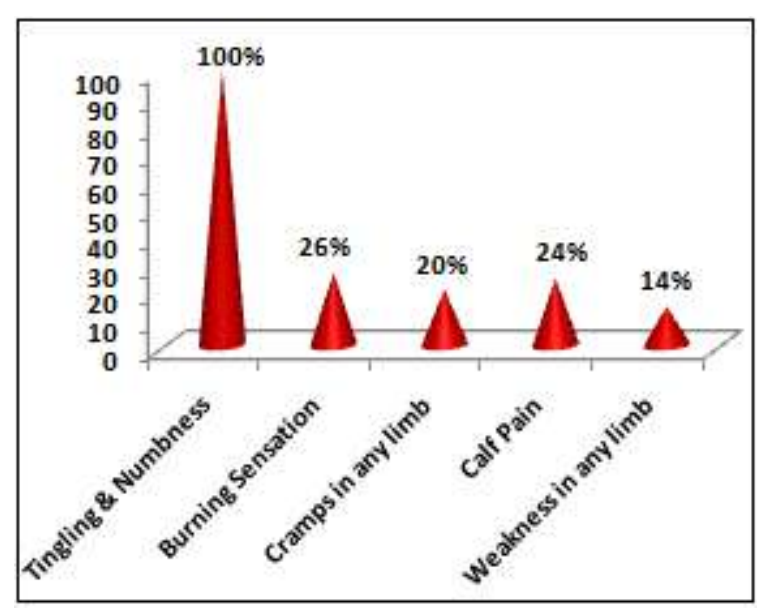

Figure 3: Symptoms Profile

\section{Conclusion}

Most common clinical presentation of diabetic neuropathy is distal symmetrical sensory neuropathy affecting mainly the lower limbs. Electrophysiological methods are more sensitive tools to detect the presence of neuropathy in diabetics especially in the patients without clinical peripheral neuropathy. Electrophysiologically, the most common type of neuropathy detected is axonal sensorimotor type which can be present even at the time of diagnosis of diabetes.

\section{References}

[1] Wild S, Roglic G, Green A, Sicree R, King H. Global prevalence of diabetes: estimates for the year 2000 and projections for 2030. Diabetes Care 2004; 27: 1047-53.

[2] E. Braunwald, A.S. Fauci, D.L. Kasper. Harrison principles of internal medicine.18th ed. McGraw-Hill, New York; 2011.

[3] P.J. Dyck, P.K. Thomas. Diabetic neuropathy (2nd Ed.)Saunders, Philadelphia, WB; 1999.

[4] L.J. Melton, F.J. Service. The prevalence by staged severity of various types of diabetic neuropathy, retinopathy, and nephropathy in a population-based cohort. The Rochester Diabetic Neuropathy Study Neurology, 43; 1993; pp.817-824.

[5] A.P. Barbosa, J.L. Medina, E.P. Ramos, H.P. Barros. Prevalence and risk factors of clinical diabetic polyneuropathy in a Portuguese primary health care population. Diabetes Metab. 27; 2001; p. 496-502.

[6] E.L. Feldman, J.W. Russell, K.A. Sullivan, D. Golovoy. New insights into the pathogenesis of diabetic neuropathy. CurrOpin Neurol. 12; 1999; pp. 553-563.

[7] American Diabetes Association: Criteria for diagnosis of diabetes, 2013; 1: 1-4.

[8] Dyck PJ, Overland CJ, Low PA, Litchy WJ, et.al Clinician vs. Neurophysician Trial Signs and symptoms versus nerve conduction studies to diagnose diabetic sensorimotor polyneuropathy: CI vs. NPhys trial. Muscle Nerve 2010; 42: 157-164.

\section{Volume 6 Issue 12, December 2017}

